\title{
Salud mental e intervenciones para padres de nińos con trastorno del espectro autista: una revisión narrativa y la relevancia de esta temática en Chile
}

\author{
Macarena Paz Fernández Suárez ${ }^{1}$, Adriana Elizabeth Espinoza Soto ${ }^{2}$ \\ Centro de Intervención Temprana (CIT-Santiago) ${ }^{1}$, Universidad de Chile ${ }^{2}$
}

\begin{abstract}
El Trastorno del Espectro Autista (TEA) afecta el neurodesarrollo durante la vida de la persona, afectando también la salud mental de los padres. Este artículo presenta una revisión narrativa de investigaciones sobre la salud mental y las intervenciones que la promueven en padres de niños entre cero y nueve años. Se revisaron bases de datos internacionales y nacionales entre los ańos 2011 y 2015, de las cuales se extrajo 18 artículos que cumplieron con los criterios establecidos. Los resultados muestran cómo las características específicas del TEA pueden afectar la salud mental de los padres crónicamente, revelando también la escasez de intervenciones psicosociales, que promuevan el bienestar emocional de estos padres y, por tanto, la necesidad de asumir un rol preventivo con esta población.

Palabras clave: Trastorno del espectro autista, padres, salud mental, intervenciones psicosociales, Chile.
\end{abstract}

Mental health and interventions for parents of children with autism spectrum disorder: A narrative review and the relevance of this topic in Chile

Autism Spectrum Disorder (ASD) affects neurodevelopment during the life of the person, also affecting the mental health of the parents. This article presents a narrative review of mental health research and interventions that promote mental health in parents of children from birth to nine years old. International and national databases between 2011 and 2015 were reviewed, of which 18 articles met the established criteria. The results show how the specific characteristics of ASD can affect the mental health of parents chronically, revealing also the scarcity of psychosocial interventions that promote the emotional well-being of these parents and, therefore, the need to assume a preventive role with this population.

Keywords: Autism spectrum disorder, parents, mental health, psychosocial interventions, Chile.

1 Licenciada en Psicología, psicóloga infanto-juvenil, Centro de Intervención Temprana (CIT-Santiago), Chile. Dirección postal: Holanda 1149, oficina 2, Providencia, Santiago, Chile. Contacto: macarena.fernandez@ug.uchile.cl ORCID: https://orcid. org/0000-0001-6819-7506

2 Doctora en Psicoterapia de la University of British Columbia, Canadá. Profesora asistente e investigadora, Departamento de Psicología, Universidad de Chile. Dirección postal: Avenida Ignacio Carrera Pinto 1045, oficina 2425, Nuñoa, Santiago, Chile. Contacto: adriana.espinoza@uchile.cl ORCID https://orcid.org/0000-0002-4039-1742 
Saúde mental e intervençóes para pais de crianças com transtorno do espectro do autismo: uma revisáo narrativa e a relevância da questáo no Chile

O Transtorno do Espectro do Autismo (TEA) afeta o neurodesenvolvimento durante a vida da pessoa, afetando também a saúde mental dos pais. Este artigo apresenta uma revisão narrativa das pesquisas sobre saúde mental e as intervençóes que a promovem em pais de crianças de zero a nove anos de idade. Com esse intuito, revisaram-se bancos de dados internacionais e nacionais entre 2011 e 2015, dos quais, 18 artigos cumpriram com os critérios estabelecidos. Os resultados mostram como as características específicas do TEA podem afetar cronicamente a saúde mental dos pais, revelando também a escassez de intervençóes psicossociais que promovem o bem-estar emocional desses pais e, portanto, a necessidade de assumir um papel preventivo nessa população.

Palavras-chave: Transtorno do espectro autista, pais, saúde mental, intervençóes psicossociais, Chile.

Santé mentale et interventions pour les parents d'enfants avec un trouble du spectre autistique: Une revue narrative et la pertinence de ce sujet au Chili

Les troubles du spectre de l'autisme (TSA), qui affectent les processus neuro-dévelopementaux au cours de la vie de une personne, peuvent compromettre également la santé mental des parents. Cet article présente un examen narratif sur la recherche à propos de la santé mentale des parents d'enfants autistes d'entre 0 et 9 ans, ainsi que sur les interventions réalisées pour la favoriser. Le contenu de bases de donées nationales et internationales a été examiné entre 2011 et 2015 , dont 18 des articles étaient conforme aux critères établis. Les résultats montrent comment les caractéristiques spécifiques des TSA peuvent affecter chroniquement la santé mentale des parents; ils révelent aussi la manque d'interventions psychosociales visant le à favoriser le bien-être émotionnel des parents, dévoilant ainsi la nécessité d'assumer un rôle préventif auprès de cette population.

Mots-clés: trouble du spectre autistique, parents, santé mentale, interventions psychosociales, Chili. 
Los Trastornos del Espectro Autista (TEA) son descritos como un complejo espectro de desórdenes del neurodesarrollo, caracterizado por déficits en la interacción social recíproca y comunicación, además de la presencia de intereses y conductas restringidas, repetitivas y estereotipadas las que se encuentran presentes a lo largo de toda la vida del individuo (Karst \& Van Hecke, 2012). Los síntomas de este trastorno destacarían por ser un continuo, observándose que algunas personas pueden mostrar compromisos más severos que otras, por lo que, al momento del diagnóstico, los profesionales clínicos debiesen considerar que tanto los síntomas como conductas variarían dependiendo de cada individuo (American Psychology Association [APA], 2013).

Respecto a la tasa de prevalencia de 12 meses para el TEA difiere entre las culturas y las naciones, y la evidencia sugiere tasas más altas de TEA en los países occidentales, como Brasil, Chile y Estados Unidos, en comparación a los países de Asia Oriental, como China, Japón, Corea o Taiwán, mostrando cifras que van entre el $6.4 \%$ y el $9.1 \%$, frente a un $0.2 \%$ y $0.8 \%$, respectivamente (Hofmann, Asnaani \& Hinton, 2010, citado en Kuusikko-Gauffi et al., 2013). En este sentido, estudios en Norteamérica, Europa y Asia han identificado que esta fluctuaría entre el 1\% y $2 \%$ (Centers for Disease Control and Prevention [CDC], 2014), estimándose una proporción de 1 niño de cada 160. Sin embargo, actuales estudios sugieren que esta cifra podría ser más alta (Organización Mundial de la Salud [OMS], 2016).

En Chile, el Ministerio de Salud (MINSAL) estableció que "no existe un registro del diagnóstico" (2011, p. 13). La única estimación con la que se cuenta se realizó el año 2007, estableciendo una cifra de alrededor de 2.156 nińos nacidos con TEA, tomando como base los 240569 nacimientos registrados ese mismo año (Ministerio de Salud [MINSAL], 2011). Otra fuente de información es el Ministerio de Educación que indica que al año 2011 existía un total de 589 estudiantes 
con este diagnóstico incorporados a programas de educación especial (MINSAL, 2011). La escasez de información actualizada sobre la prevalencia de este trastorno en niños y adolescentes, hace difícil estimar recursos, elaborar programas e intervenciones lo que anticipa un problema de salud pública para la atención de estos niños y sus padres. A lo anterior se suma que el gasto en salud en Chile sigue siendo inferior al de la mayoría de los países de la Organización para la Cooperación y el Desarrollo Económicos [OCDE]. Lo que implica que menos del 50\% del gasto total en salud es financiado con medios públicos y una gran parte es pagada directamente por las familias (OCDE, 2015).

En relación con las investigaciones sobre este trastorno se puede observar que en una primera etapa, el foco de las investigaciones se centraban en estudiar, evaluar y proponer intervenciones específicamente en pacientes con TEA, dejando de lado a los padres y sus familias, o solo incluyéndolas como un apoyo para el mejoramiento del niño y/o adolescente (Da Paz \& Wallander, 2016; Geoffray, Thevenet \& Georgieff, 2016; Karst \& Van Hecke, 2012; Agency for Healthcare Research and Quality [AHRQ], 2011). Sin embargo, en las últimas décadas y debido al aumento de interés sobre este trastorno, la salud mental de los padres de estos pacientes se ha vuelto otro foco de atención. En este sentido, y a partir de la información encontrada en la literatura, los grandes ejes de estudio internacional sobre salud mental en estos padres se han focalizado principalmente en medir estrés, ansiedad y depresión (Dababnah \& Parish, 2015; Golfenshtein, Srulovici y Medoff-Cooper, 2015; Gomes et al., 2015; Lee, 2013; Ooi, Ong, Jacob \& Khan, 2016; Whitmore, 2016; Bonis \& Sawin, 2016) por lo que, en esta revisión, esos serán los conceptos a considerar. No obstante, además de lo anterior, cabe destacar que la OMS (s.f.) señala dentro de su definición de salud mental, el "completo bienestar físico, mental y social”, por lo que se decidió no considerar exclusivamente el área de la psicopatología, sino también incorporar el concepto de bienestar psicológico dentro de esta revisión, así como las intervenciones psicológicas y psicosociales para esta población.

El propósito de esta revisión narrativa es proporcionar un panorama general que permita caracterizar los estudios enfocados en la salud 
mental de padres de niños con TEA, específicamente estrés, ansiedad, depresión y bienestar psicológico, así como el tipo de intervenciones que se implementan con esta población, realizando un análisis descriptivo de los resultados de los estudios seleccionados. Para esto se revisaron diversas bases de datos y fuentes de información tanto internacionales como nacionales publicadas entre los años 2011 y 2015 en inglés y español. Las preguntas que guiaron esta revisión fueron: (a) ¿Cuáles han sido las características y los resultados de las investigaciones que se han realizado respecto a la salud mental en padres de hijos con trastorno del espectro autista publicadas entre los años 2011 y 2015, tanto a nivel internacional como nacional?; y (b) ¿Cuáles han sido las características y los resultados de las investigaciones que han realizado intervenciones que buscan promover la salud mental en padres de hijos con trastorno del espectro autista publicadas entre los años 2011 y 2015, tanto a nivel internacional como nacional?

\section{Salud mental en padres de niños con TEA}

Los padres de un hijo con autismo, a la vez que cumplen la función primordial del cuidado, deben hacer frente a una serie de dificultades, tanto en el campo emocional como práctico (Cabezas, 2001). $\mathrm{Al}$ recibir el diagnóstico de TEA, se inicia un proceso largo y doloroso, el cual no estará exento de dudas, miedos, angustia y cuestionamientos sobre cómo se convive y trata a un niño con esas características. A las demandas que ya significan la crianza de un nińo con desarrollo normal, a los padres de hijos con TEA, se añaden las relacionadas con las condiciones del trastorno de su hijo. Estas demandas adicionales pueden generar aumento de estrés, que pueden traer cambios a nivel psicológico, disminución en la respuesta del sistema inmune y riesgo de enfermedades (Whitmore, 2016). Padres que sufren de estrés mostrarían más probabilidades de presentar dificultades en las relaciones y en la crianza; lo cual puede incluso tener un impacto negativo en el nińo, como riesgo de abuso o negligencia (Whitmore, 2016).

Entre los resultados de un metaanálisis realizado por Ooi y colaboradores (2016), una categoría de análisis generada dentro de su revisión 
era la de "niño difícil", en la cual identificaron que, las rabietas incontrolables e impredecibles y las conductas agresivas, serían las que causan mayor estrés en los padres de hijos con TEA. Sumado a esto, los otros ejes temáticos muestran que el impacto de tener un hijo con TEA también afecta en áreas como la salud, economía, profesión y/o tiempo de ocio; lo que contribuiría a aumentar los niveles de estrés percibido.

Asimismo, los padres de los niños con problemas del desarrollo enfrentan retos considerables en su vida cotidiana y se sabe que experimentan niveles elevados de ansiedad (Hamlyn-Wright et al., 2007; Singer et al., 2007, citado en Uljarevic, Carrintong \& Leekam, 2016). La relación entre los síntomas de ansiedad experimentados por los padres y los experimentados por sus hijos con TEA solo se ha explorado recientemente (Conner, Maddox \& White, 2013). Sin embargo, existiría evidencia de que los padres de hijos con TEA serían más propensos a tener trastornos afectivos, incluyendo trastornos de ansiedad, en comparación con las normas de población (Uljarevic et al., 2012, citado en Conner et al., 2013). Se ha encontrado, además, que algunos tipos de sintomatología ansiosa más recurrente en estos padres serían, por nombrar algunas, manifestaciones como pensamientos catastróficos, quejas somáticas como palpitaciones fuertes del corazón, sudoración de palmas, entre otras (Al-Farsi, Al-Farsi, Al-Sharbati \& Al-Adawi, 2016).

Respecto a la depresión, las investigaciones han demostrado que padres de hijos con TEA presentan alto riesgo de desarrollar depresión, en comparación a padres de hijos sin el trastorno (Bekhet \& Zauszniewski, 2013; Snow \& Donnelly, 2016; Van Steijn, Oerlemans, Van Aken, Buitelaar \& Rommelse, 2016; Zablotsky, Anderson \& Law, 2013). En concordancia con esto, se ha observado que nińos y adolescentes con TEA que experimentan depresión pueden aumentar el riesgo, a su vez, de depresión en sus cuidadores (Bekhet, 2014). En un estudio de Zhou y Yi (2014) padres de nińos con TEA reportaron emociones negativas intensas como ansiedad, desesperanza, rabia e impotencia, sumado a la percepción de que sus propias emociones parecían influenciar las reacciones emocionales y síntomas de sus hijos. En una sistematización de la literatura realizada por AlHorany, Younis, Bataineh y Hassan (2013), 
se buscó revisar cuáles serían los factores que afectarían en la depresión de madres de hijos con TEA, llegando a la conclusión de que no existían pruebas concluyentes de esto. Sin embargo, encontraron una cantidad sustancial de artículos que documentan que ser padre de un niño autista muestra un amplio espectro de sentimientos y problemas, por ejemplo, gran estrés, problemas de salud física, sentimientos de culpa, cólera, problemas conyugales, entre otros. Estos podrían asociarse a un posible desarrollo de sintomatología depresiva. Finalmente, y como lo han identificado los estudios de Romero-Martínez et al. $(2016,2017)$ el cuidado de estos niños por un período extendido en el tiempo tiene efectos crónicos en la salud mental de los padres cuidadores. Estos investigadores han concluido que tanto hombres como mujeres presentan una memoria verbal y declarativa empobrecida así como mayores problemas con la memoria retrospectiva y prospectiva (Romero-Martínez et al. 2016, 2017). Estos hallazgos relevan también la importancia de estudiar la disminución de habilidades cognitivas en esta población producida por los altos niveles de estrés a los que han estado sometidos durante su vida como cuidadores de hijos con TEA.

En relación con el bienestar de las familias de individuos con TEA, este raramente ocupa un lugar central en la investigación y en las políticas públicas (Cridland, Jones, Magee \& Caputi, 2014). Desde la intervención clínica, la mayoría de los sistemas de atención se organizan para satisfacer las necesidades de los pacientes individuales, en donde las necesidades de los cuidadores familiares rara vez se abordan (Karst \& Van Hecke, 2012; Romero-Martínez et al., 2017). En una revisión sistemática realizada por Da Paz y Wallander (2017), encontraron que algunas de las variables asociadas al bienestar en padres de hijos con TEA podrían estar relacionadas con la severidad de síntomas autistas en el hijo, el apoyo social recibido, los estilos de afrontamiento, resiliencia, entre otros (Bekhet, Johnson \& Zauszniewski, 2012).

Finalmente, el incipiente interés por la salud mental de los padres de niños con TEA se demuestra en la propuesta de diversas intervenciones que apuntarían a mejorar su salud mental (Da Paz \& Wallander, 2016; Kars \& Van Hecke, 2012; Kogan, Barbui, Smith, Yasamy \& 
Servili, 2014; Romero-Martínez, 2017), las cuales incluyen terapia cognitivo conductual, de expresión a través de la escritura, mindfulness y estrategias basadas en psicología positiva y técnicas de relajación (Da Paz \& Wallander, 2016) y que pueden poseer modalidad individual como grupal. Lo anterior es de gran relevancia pues, las intervenciones tempranas dirigidas a favorecer la salud mental de los padres, también facilitan cambios positivos en los comportamientos relacionados con el TEA del niño. Consecuentemente, reducir el estrés de los padres con una intervención temprana puede facilitar el funcionamiento familiar mediante el restablecimiento del equilibrio y, por tanto, moderar y reducir los efectos de los problemas de comportamiento (Cachia, Anderson \& Moore, 2016; Hayes \& Watson, 2013; Romero-Martínez, 2017). Asimismo, es importante considerar que este trastorno afecta la salud mental de los padres a largo plazo incluyendo problemas cognitivos $y$ en particular a nivel de empobrecimiento de la memoria afectando el entorno familiar, su capacidad y la calidad del cuidado que puedan otorgar a sus hijos a largo plazo (Romero-Martínez et al., 2016, 2017).

Dados los antecedentes presentados respecto a la salud mental, el bienestar psicológico y las intervenciones para padres de hijos con TEA es que se realiza la presente revisión teniendo como objetivo transmitir conocimiento que genere un diálogo entre los profesionales de la salud y la salud mental en Chile, con el fin de visibilizar y promover el bienestar y la salud mental de estos padres, pues son ellos quienes se ven altamente exigidos ante las demandas asociadas al trastorno, volviéndose así un grupo vulnerable de sufrir algún trastorno psicológico, como ansiedad, depresión y estrés, entre otros, pudiendo afectar su calidad de vida y la de su entorno familiar a corto mediano y a largo plazo.

\section{Método}

Se realizó una revisión narrativa, la cual se caracteriza por describir y discutir el desarrollo o "estado del arte" sobre un tema determinado, desde el punto de vista teórico o contextual, el cual tiene un 
papel fundamental para la educación continua, pues permite al lector adquirir y actualizar el conocimiento sobre una temática específica en un corto espacio y tiempo (Rother, 2007).

La búsqueda bibliográfica se concentró en las bases de datos ISI Web of Science, Scopus, Pubmed, Redalyc, SciELO, Revista de Psicología Chilena, Revista Médica de Chile y Google Scholar. Se utilizaron las palabras clave "autism spectrum disorder", "mental health", "parents" e "intervention". Lo anterior se combinó con otras palabras clave por separado: "mother", "father", "stress", "depression”, "anxiety", "wellbeing" y "psychological intervention". Estas mismas palabras claves fueron usadas para la búsqueda en español.

Además de estos criterios de búsqueda, se emplearon los siguientes criterios de inclusión, considerando tanto estudios cualitativos como cuantitativos: 1) los estudios debían ser exclusivos de padres de hijos diagnosticados con TEA, incluyendo en la evaluación y/o intervención a ambos padres o por separado; 2) la edad de estos hijos debía estar entre los cero a nueve ańos, siendo mencionado dentro de las características de la muestra. Esto se debe a que algunas investigaciones incluyen padres de niños con TEA y padres de adultos con TEA indistintamente; 3) las investigaciones debían evaluar la salud mental de estos padres con posterioridad al diagnóstico de TEA en sus hijos, y no identificar psicopatología anterior a la confirmación diagnóstica, dado que existen investigaciones que evalúan salud mental en padres previo a la confirmación de TEA en su hijo intentando correlacionar la psicopatología en los padres y riesgo de autismo (Croen, Grether, Yoshida, Odouli \& Hendrick, 2011; Rai, Lee, Dalman, Golding, Lewis \& Magnusson, 2013; Roberts, Koenen, Lyall, Ascherio \& Weisskopf, 2014); 4) los estudios debían considerar exclusivamente a padres de niños con TEA, sin incluir otras patologías, como por ejemplo, otros trastornos del neurodesarrollo; 5) respecto al concepto salud mental, se incluyeron estudios que evaluaron y/o se enfocaron en medir: ansiedad, depresión, estrés y bienestar psicológico en padres de niños con TEA, lo cual debía quedar explicitado en el título, palabras clave o resumen; y 6) en cuanto a las investigaciones sobre intervenciones que promovieran 
la salud mental de estos padres, se seleccionaron aquellas que realizaron intervenciones o programas que especificaban dentro de sus objetivos o intereses el mejorar o promover la salud mental de los padres, lo que debía quedar explicitado en el título, palabras claves o resumen.

\section{Proceso de selección de artículos}

Se realizó la búsqueda con las palabras clave y ańos establecidos, lo que arrojó 1563 artículos para ser candidatos a revisión. De estos, 76 evaluaban el concepto de salud mental e intervenciones con padres de niños con TEA. Tras esto, de cada artículo se revisó el resumen, definición de conceptos y objetivos, por lo que se redujo a 58 las posibles investigaciones para ser incluidas en esta revisión. Finalmente, tras la aplicación exhaustiva de los criterios de inclusión se determinó que 18 de ellos cumplían con los requisitos para ser incluidos y estudiados en esta revisión narrativa.

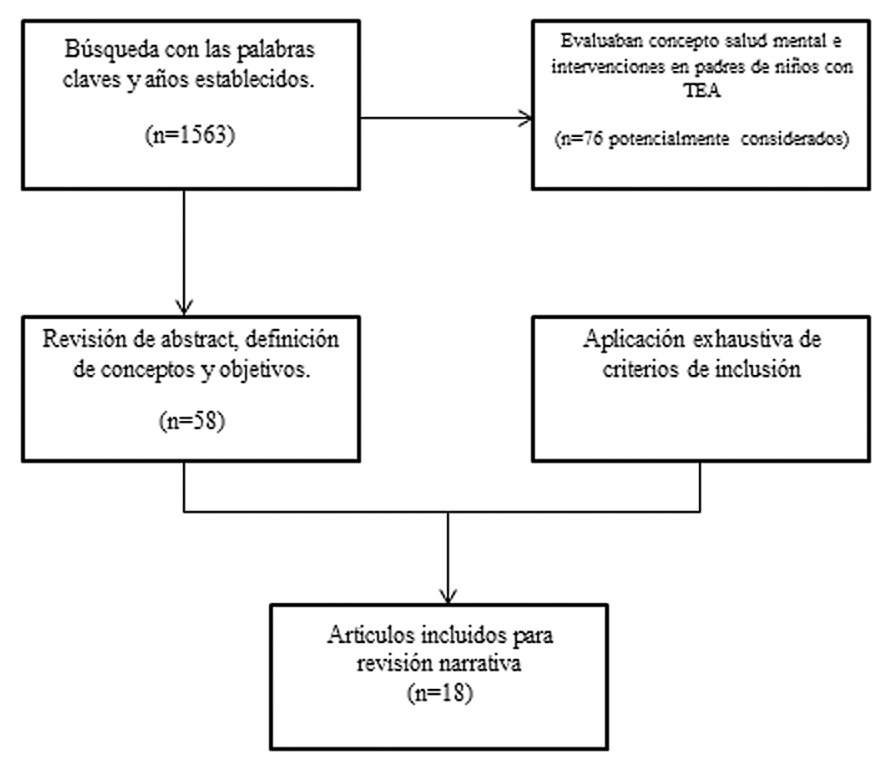

Diagrama de selección de artículos 


\section{Resultados}

Los resultados de esta revisión se presentarán siguiendo los tres conceptos encontrados en la literatura asociados a salud mental (estrés, ansiedad y depresión) seguidos por bienestar psicológico e intervenciones que promuevan la salud mental de estos padres.

De los 1563 artículos arrojados tras las búsquedas en las diferentes bases de datos, 76 de ellos fueron potencialmente considerados para esta revisión. De esos, 58 fueron excluidos, principalmente por no cumplir los criterios de inclusión. Finalmente, un número de 18 artículos fueron seleccionados para esta revisión. Cabe destacar que, a nivel nacional, no se encontraron artículos que cumplieran con los criterios de inclusión de esta revisión. A pesar de esto, es relevante mencionar que existen diversos estudios chilenos sobre TEA, encontrándose el foco en aspectos biológicos y/o cognitivos del trastorno, en individuos de diversas edades (ver Aguaded \& Almeida, 2016; Irarrázaval, Brokering \& Murillo, 2005; Morales, Solovieva, Lázaro, Quintanar \& Machinskaya, 2014; Quijada, 2008; Zalaquett, Schönstedt, Angeli, Herrera \& Moyano, 2015). Estos estudios demuestran que, de alguna forma, siguen la tendencia internacional que se ha registrado en donde las investigaciones en una primera etapa, se centraban en estudiar, evaluar el trastorno y generar intervenciones y estrategias exclusivamente para niños con TEA, excluyendo a sus padres.

También en la búsqueda nacional aparecieron tesis universitarias que poseen diversos focos de estudio, metodología y aproximación a la temática, como intervenciones desde el arte y la música a niños con TEA (Fernández, 2016; Miranda, 2007; Saa, 2014); evaluación del área cognitiva en niños y adolescentes con el trastorno (Berrios, Catalán, Muñoz, Maureira \& Santibañez, 2012; Moya, 2016; Soto, 2007); y aproximaciones teóricas desde distintos enfoques de la psicología, tales como el psicoanálisis y el constructivismo evolutivo (Carrera, 2012; Llantén \& Valencia, 2001; Puglisevich, 2014), entre otras. Las tesis que destacan por mostrar mayor concordancia con esta revisión, son dos que se centran en el impacto familiar que genera el tener un hijo con 
TEA en la familia, desde un enfoque cualitativo (Hernández, 2008; Tobar, 2005) y otra sobre estrés parental en padres de niños con TEA desde la metodología cuantitativa (Tereucán \& Treimún, 2016). Sin embargo, tampoco cumplían los criterios para ser incluidos en esta revisión.

En términos de las características, todos los artículos de carácter internacional utilizaron metodología cuantitativa para la recolección de los datos. De estas investigaciones, cinco se centraban en el concepto salud mental a nivel general, es decir, medían ansiedad y/o depresión y/o estrés, y 12 en alguna variable específica de estas. Sobre intervenciones, se encontró un artículo que buscaba promover la salud mental. Respecto a los ańos de las publicaciones, 11 artículos (61.1\%) fueron publicados durante los ańos 2013-2014, cuatro artículos entre 2011$2012(22.2 \%)$, seguido por tres publicaciones durante 2015-2016 (16.6\%). Dentro de las características de las investigaciones, se observa que la mayoría de los estudios fueron realizados en Estados Unidos (33.3\%), seguido por Australia (22.2\%), Canadá (16.6\%), Reino Unido (16,6\%), Italia (5.5\%) e Irán (5.5\%). En cuanto a los focos de estudio, la mitad se centraban en las madres y la otra mitad en ambos padres, no encontrándose artículos que evaluaran exclusivamente a los padres. La Tabla 1 proporciona información sobre los artículos seleccionados para esta revisión. 
Salud mental e intervenciones para padres de niños con TEA / Fernández Suárez, Espinoza Soto

\section{Tabla 1}

\section{Caracteristicas descriptivas de investigaciones sobre salud mental en padres de hijos con TEA}

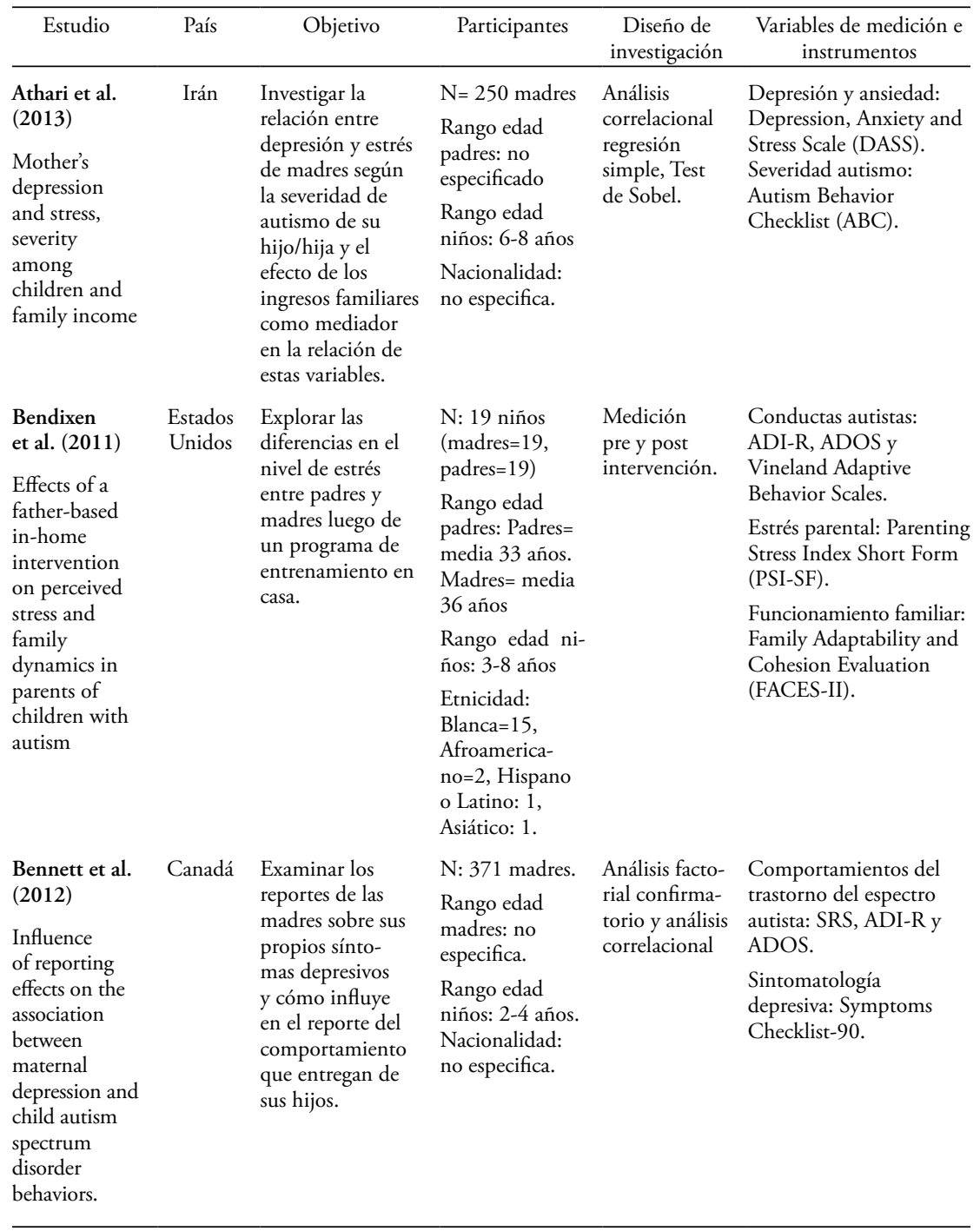


Tabla 1 (continuación)

\begin{tabular}{|c|c|c|c|c|c|}
\hline Estudio & País & Objetivo & Participantes & $\begin{array}{c}\text { Diseño de } \\
\text { investigación }\end{array}$ & $\begin{array}{l}\text { Variables de medición e } \\
\text { instrumentos }\end{array}$ \\
\hline \multirow{8}{*}{$\begin{array}{l}\text { Giallo et al. } \\
(2013) \\
\text { Fatigue, } \\
\text { wellbeing } \\
\text { and parental } \\
\text { self-efficacy } \\
\text { in mothers } \\
\text { of children } \\
\text { with autism } \\
\text { spectrum } \\
\text { disorder. }\end{array}$} & \multirow[t]{8}{*}{ Australia } & \multirow{8}{*}{$\begin{array}{l}\text { Examinar la } \\
\text { experiencia de } \\
\text { fatiga de padres } \\
\text { de niños con TEA } \\
\text { y su relación con } \\
\text { el bienestar y } \\
\text { crianza. }\end{array}$} & \multirow{8}{*}{$\begin{array}{l}\text { N: } 50 \text { madres. } \\
\text { Rango edad } \\
\text { madres: } \\
\text { 21-46 años. } \\
\text { Rango edad niños: } \\
\text { 2-5 ańos. } \\
\text { Nacionalidad: } \\
\text { Australia=46, } \\
\text { Otro=3, } \\
\text { No reportado=1. }\end{array}$} & \multirow{8}{*}{$\begin{array}{l}\text { Análisis } \\
\text { correlacional. } \\
\text { Análisis } \\
\text { factorial } \\
\text { confirmatorio, } \\
\text { análisis de } \\
\text { regresión de } \\
\text { múltiples } \\
\text { variables }\end{array}$} & $\begin{array}{l}\text { Fatiga: Fatigue Assessment } \\
\text { Scale (Adapted FAS). }\end{array}$ \\
\hline & & & & & $\begin{array}{l}\text { Estados emocionales } \\
\text { negativos: Depression, } \\
\text { Anxiety and Stress Scale } \\
21 \text { (DASS). }\end{array}$ \\
\hline & & & & & $\begin{array}{l}\text { Autoeficacia parental: The } \\
\text { Parenting Sense of Com- } \\
\text { petence Scale (PSOC). }\end{array}$ \\
\hline & & & & & $\begin{array}{l}\text { Problemas conductuales } \\
\text { y emocionales en niños } \\
\text { con discapacidad: } \\
\text { Developmental Behavior } \\
\text { Checklist P24 (DBC-P). }\end{array}$ \\
\hline & & & & & $\begin{array}{l}\text { Evaluar calidad del sueńo: } \\
\text { Pittsburgh Sleep Quality } \\
\text { Index (PSQI). }\end{array}$ \\
\hline & & & & & $\begin{array}{l}\text { Necesidades sociales y } \\
\text { apoyo social: Parent Social } \\
\text { Support Index }\end{array}$ \\
\hline & & & & & $\begin{array}{l}\text { Comportamiento de } \\
\text { la salud: The Health } \\
\text { Behavior Scale. }\end{array}$ \\
\hline & & & & & $\begin{array}{l}\text { Información } \\
\text { socioeconómica: Socio- } \\
\text { economic Indexes } \\
\text { for Areas Index of } \\
\text { Relative Socioeconomic } \\
\text { Disadvantages. }\end{array}$ \\
\hline \multirow{5}{*}{$\begin{array}{l}\text { Jellett et al. } \\
(2015) \\
\text { Family } \\
\text { functioning } \\
\text { and behavior } \\
\text { problems } \\
\text { in children } \\
\text { with Autism } \\
\text { Spectrum } \\
\text { Disorders: } \\
\text { The } \\
\text { mediating } \\
\text { role of parent } \\
\text { mental } \\
\text { health. }\end{array}$} & \multirow[t]{5}{*}{ Australia } & \multirow{5}{*}{$\begin{array}{l}\text { Explorar la } \\
\text { relación entre } \\
\text { los problemas de } \\
\text { comportamiento } \\
\text { del niño con } \\
\text { TEA y el } \\
\text { funcionamiento } \\
\text { familiar, } \\
\text { estudiando como } \\
\text { mediadores las } \\
\text { dificultades en la } \\
\text { salud mental de } \\
\text { los padres (estrés, } \\
\text { fatiga y síntomas } \\
\text { depresivos). }\end{array}$} & \multirow{5}{*}{$\begin{array}{l}\text { N: } 97 \\
\text { (Madres=88, } \\
\text { Padres=9). } \\
\text { Rango edad } \\
\text { padres: media = } \\
36 \text { ańos. } \\
\text { Rango edad } \\
\text { niños:16-71 } \\
\text { meses. } \\
\text { Tipo familiar: } \\
\text { Uniparental= } 10 \text {, } \\
\text { En pareja: } 87 . \\
\text { Nacionalidad: } \\
\text { Australia=76, } \\
\text { Otro país= } 21 .\end{array}$} & \multirow[t]{5}{*}{$\begin{array}{l}\text { Análisis } \\
\text { correlacional, } \\
\text { generación de } \\
\text { modelo, }\end{array}$} & $\begin{array}{l}\text { Estrés y síntomas depresi- } \\
\text { vos en padres: Depression, } \\
\text { Anxiety and Stress Scale } \\
(\text { DASS-21). }\end{array}$ \\
\hline & & & & & $\begin{array}{l}\text { Fatiga: Fatigue Assessment } \\
\text { Scale (FAS) }\end{array}$ \\
\hline & & & & & $\begin{array}{l}\text { Comportamiento del } \\
\text { niño: Developmental } \\
\text { Behavior Checklist- } \\
\text { Parent Short Form } \\
\text { (DBC-P24). }\end{array}$ \\
\hline & & & & & $\begin{array}{l}\text { Funcionamiento familiar: } \\
\text { General Functioning } \\
\text { Scale (FAD-GF). }\end{array}$ \\
\hline & & & & & $\begin{array}{l}\text { Información familiar y } \\
\text { demográfica. }\end{array}$ \\
\hline
\end{tabular}


Salud mental e intervenciones para padres de niños con TEA / Fernández Suárez, Espinoza Soto

Tabla 1 (continuación)

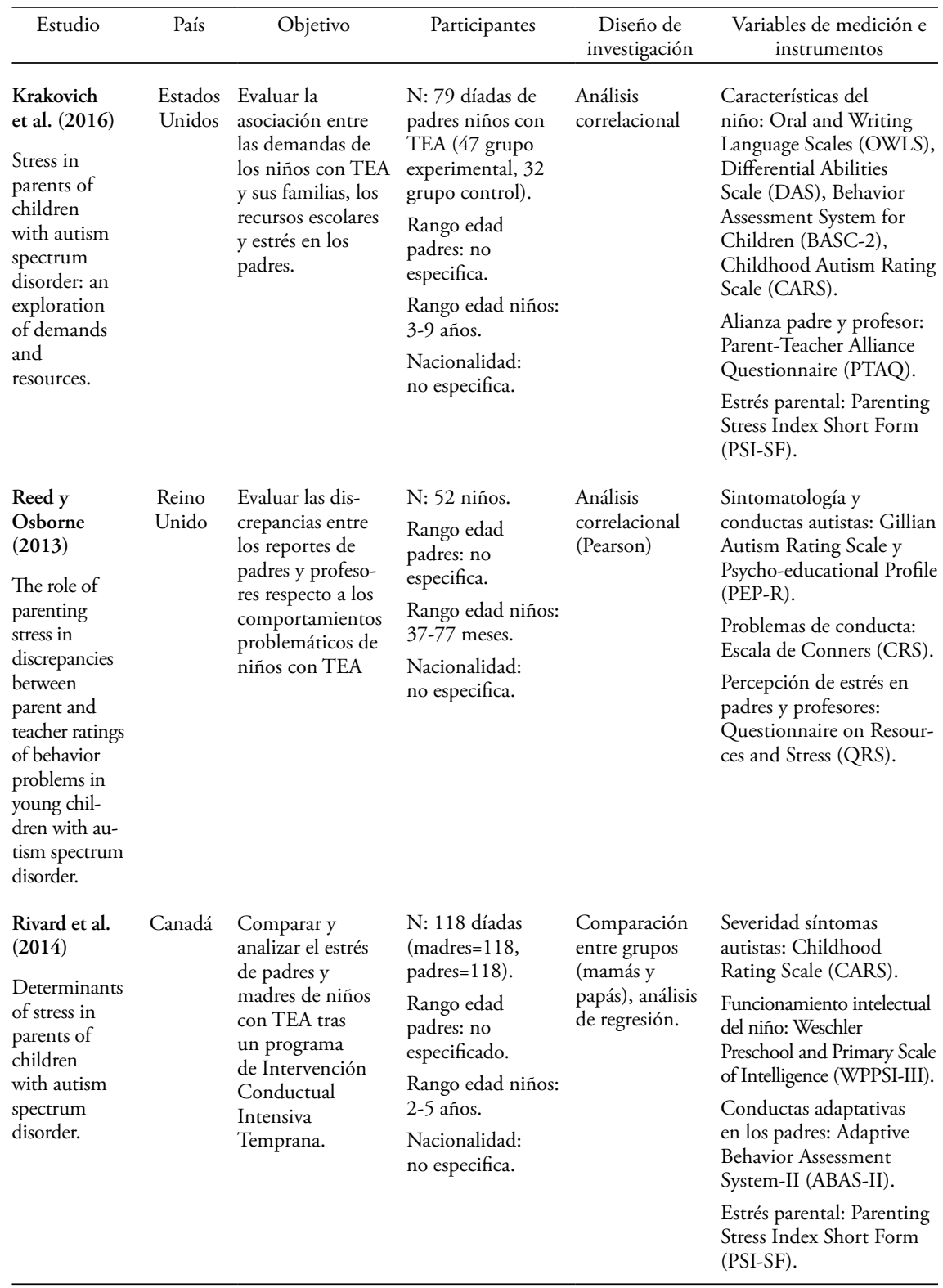


Tabla 1 (continuación)

\begin{tabular}{|c|c|c|c|c|c|}
\hline Estudio & País & Objetivo & Participantes & $\begin{array}{c}\text { Diseño de } \\
\text { investigación }\end{array}$ & $\begin{array}{l}\text { Variables de medición e } \\
\text { instrumentos }\end{array}$ \\
\hline $\begin{array}{l}\text { Seymour et al. } \\
\text { (2013) } \\
\text { Fatigue, stress } \\
\text { and coping } \\
\text { in mothers of } \\
\text { children with } \\
\text { an autism } \\
\text { spectrum } \\
\text { disorder. }\end{array}$ & Australia & $\begin{array}{l}\text { Investigar la } \\
\text { influencia de la } \\
\text { fatiga materna y } \\
\text { afrontamiento en } \\
\text { la relación entre } \\
\text { los problemas de } \\
\text { comportamiento } \\
\text { del nińo con } \\
\text { TEA y el estrés } \\
\text { maternal. }\end{array}$ & $\begin{array}{l}\text { N: } 65 \text { madres. } \\
\text { Rango edad } \\
\text { madres: media= } \\
36 \text { años. } \\
\text { Rango edad niños: } \\
2-5 \text { años. } \\
\text { Nacionalidad: } \\
\text { Australia }=55, \\
\text { Otros }=10 .\end{array}$ & $\begin{array}{l}\text { Análisis } \\
\text { correlacional, } \\
\text { generación de } \\
\text { modelo. }\end{array}$ & $\begin{array}{l}\text { Información } \\
\text { demográfica: } \\
\text { Demographic and } \\
\text { Family Background } \\
\text { Questionnaire. } \\
\text { Problemas de } \\
\text { comportamiento en } \\
\text { niños con discapacidad: } \\
\text { The Brief Developmental } \\
\text { Behavior Checklist-P24 } \\
\text { (DBC-P24). } \\
\text { Estrés: The Depression, } \\
\text { Anxiety and Stress } \\
\text { scale-21 (DASS-21). Se } \\
\text { utilizó solo la subescala } \\
\text { de Estrés. } \\
\text { Fatiga: Fatigue } \\
\text { Assessment Scale (FAS). } \\
\text { Afrontamiento en padres: } \\
\text { The Brief COPE. }\end{array}$ \\
\hline $\begin{array}{l}\text { Strauss et al. } \\
\text { (2012) } \\
\text { Parent } \\
\text { Inclusion in } \\
\text { Early Intensive } \\
\text { Behavioral } \\
\text { Intervention: } \\
\text { The Influence } \\
\text { of Parental } \\
\text { Stress, Parent } \\
\text { Treatment } \\
\text { Fidelity } \\
\text { and Parent- } \\
\text { Mediated } \\
\text { Generalization } \\
\text { of Behavior } \\
\text { Targets } \\
\text { on Child } \\
\text { Outcomes. }\end{array}$ & Italia & $\begin{array}{l}\text { Evaluar la } \\
\text { asociación } \\
\text { entre el estrés } \\
\text { de los padres y } \\
\text { la fidelidad al } \\
\text { tratamiento. }\end{array}$ & $\begin{array}{l}\text { N: } 44 \text { padres } \\
\text { (Grupo } \\
\text { experimental=24, } \\
\text { Grupo } \\
\text { control=20). } \\
\text { Rango edad } \\
\text { padres: No } \\
\text { específica. } \\
\text { Rango edad niños: } \\
\text { 26-81 meses. } \\
\text { Nacionalidad: No } \\
\text { especifica. }\end{array}$ & $\begin{array}{l}\text { Medición } \\
\text { intra y entre } \\
\text { grupos } \\
\text { (intervenido } \\
\text { vs ecléctico) }\end{array}$ & $\begin{array}{l}\text { Variables del niño: } \\
\text { Autism Diagnostic } \\
\text { Observation Schedule } \\
\text { (ADOS), The Griffith } \\
\text { Mental Developmental } \\
\text { Scales for Ages } 2 \text { to } \\
8 \text { (GMDS-ER 2-8), } \\
\text { Vineland Adaptive } \\
\text { Behavior Scales- } \\
\text { Interview Edition } \\
\text { (VABS), McArthur } \\
\text { Communication } \\
\text { Developmental } \\
\text { Inventories (CDI), } \\
\text { Frequencies of Child's } \\
\text { Challenging Behavior. } \\
\text { Variables padres: Parental } \\
\text { Stress Index (PSI-SF), } \\
\text { Parent Treatment } \\
\text { Fidelity. }\end{array}$ \\
\hline
\end{tabular}


Salud mental e intervenciones para padres de niños con TEA / Fernández Suárez, Espinoza Soto

Tabla 1 (continuación)

\begin{tabular}{|c|c|c|c|c|c|}
\hline Estudio & País & Objetivo & Participantes & $\begin{array}{c}\text { Diseńo de } \\
\text { investigación }\end{array}$ & $\begin{array}{c}\text { Variables de medición e } \\
\text { instrumentos }\end{array}$ \\
\hline $\begin{array}{l}\text { Tellegen y } \\
\text { Sanders (2014) } \\
\text { A Randomized } \\
\text { Controlled Trial } \\
\text { Evaluating a } \\
\text { Brief Parenting } \\
\text { Program with } \\
\text { Children } \\
\text { with Autism } \\
\text { Spectrum } \\
\text { Disorders. }\end{array}$ & Australia & $\begin{array}{l}\text { Evaluar la } \\
\text { eficacia de } \\
\text { Primary Pie Step } \\
\text { Stones Triple } \\
\text { P e identificar } \\
\text { cambios en } \\
\text { estrés, ansiedad } \\
\text { y depresión en } \\
\text { padres de niños } \\
\text { con TEA. }\end{array}$ & $\begin{array}{l}\text { N: } 64 \text { padres } \\
\text { (Grupo } \\
\text { experimental: } \\
\text { Madres=34, } \\
\text { Padres=1; } \\
\text { Grupo control: } \\
\text { Madres=27, } \\
\text { Padres=2). } \\
\text { Rango edad } \\
\text { padres: } 36-38 \\
\text { ańos. } \\
\text { Rango edad } \\
\text { niños: } 2-9 \text { años. } \\
\text { Nacionalidad: } \\
\text { No especifica. }\end{array}$ & $\begin{array}{l}\text { Ensayo clínico } \\
\text { aleatorizado. } \\
\text { Grupo } \\
\text { intervenido } \\
\text { vs. Cuidado } \\
\text { habitual. } \\
\text { Se evaluó } \\
\text { preintervención, } \\
\text { postintervención } \\
\text { y seguimiento de } \\
6 \text { meses }\end{array}$ & $\begin{array}{l}\text { Información } \\
\text { demográfica: } \\
\text { Family Background } \\
\text { Questionnaire (FBQ). } \\
\text { Problemas de } \\
\text { comportamiento en } \\
\text { el niño: The Eyberg } \\
\text { Child Behavior } \\
\text { Inventory (ECBI). } \\
\text { Crianza disfuncional: } \\
\text { The Parenting Scale (PS). } \\
\text { Confianza en los } \\
\text { padres: The Parenting } \\
\text { Tasks Checklist (PTC). } \\
\text { Ajuste parental: The } \\
\text { Depression, Anxiety } \\
\text { and Stress Scales-21 } \\
\text { (DASS-21). } \\
\text { Estrés parental: } \\
\text { Parental Stress Scale } \\
\text { (PSS). } \\
\text { Ajuste en la relación } \\
\text { marital: Parent } \\
\text { Problem Checklist } \\
\text { (PPC) y Relationship } \\
\text { Quality Index (RQI). } \\
\text { Éxito del Programa: } \\
\text { The Goal Achievement } \\
\text { Scales (GAS) y The } \\
\text { Client Satisfaction } \\
\text { Questionnaire (CSQ). }\end{array}$ \\
\hline $\begin{array}{l}\text { Totsika et al. } \\
\text { (2013) } \\
\text { Is there a } \\
\text { bidirectional } \\
\text { relationship } \\
\text { between maternal } \\
\text { well-being and } \\
\text { child behavior } \\
\text { problems in } \\
\text { autism spectrum } \\
\text { disorders? } \\
\text { Longitudinal } \\
\text { analysis of a } \\
\text { population- } \\
\text { defined simple of } \\
\text { Young children. }\end{array}$ & Reino Unido & $\begin{array}{l}\text { Examinar si la } \\
\text { relación entre } \\
\text { el bienestar } \\
\text { psicológico } \\
\text { materno y los } \\
\text { problemas de } \\
\text { comportamiento } \\
\text { en nin̄os } \\
\text { con TEA es } \\
\text { bidireccional. }\end{array}$ & $\begin{array}{l}\text { N: } 132 \text { niños. } \\
\text { (Madres=99\%, } \\
\text { Padres=1\%). } \\
\text { Rango edad } \\
\text { padres: } 32-34 \\
\text { años. } \\
\text { Edad niños: } 5 \\
\text { años. } \\
\text { Nacionalidad: } \\
\text { Reino Unido } \\
\text { principalmente } \\
\text { (no especifica). }\end{array}$ & $\begin{array}{l}\text { Generación } \\
\text { de modelo, } \\
\text { correlaciones. }\end{array}$ & $\begin{array}{l}\text { Problemas de conducta } \\
\text { del niño: The Strengths } \\
\text { and Difficulties } \\
\text { Questionnaire } \\
\text { (SDQ), The Revised } \\
\text { Infant Temperament } \\
\text { Questionnaire (RITQ). } \\
\text { Bienestar materno: } \\
\text { K6, The Malaise } \\
\text { Inventory, The Physical } \\
\text { Component Summary } \\
\text { of the SF-8. }\end{array}$ \\
\hline
\end{tabular}


Tabla 1 (continuación)

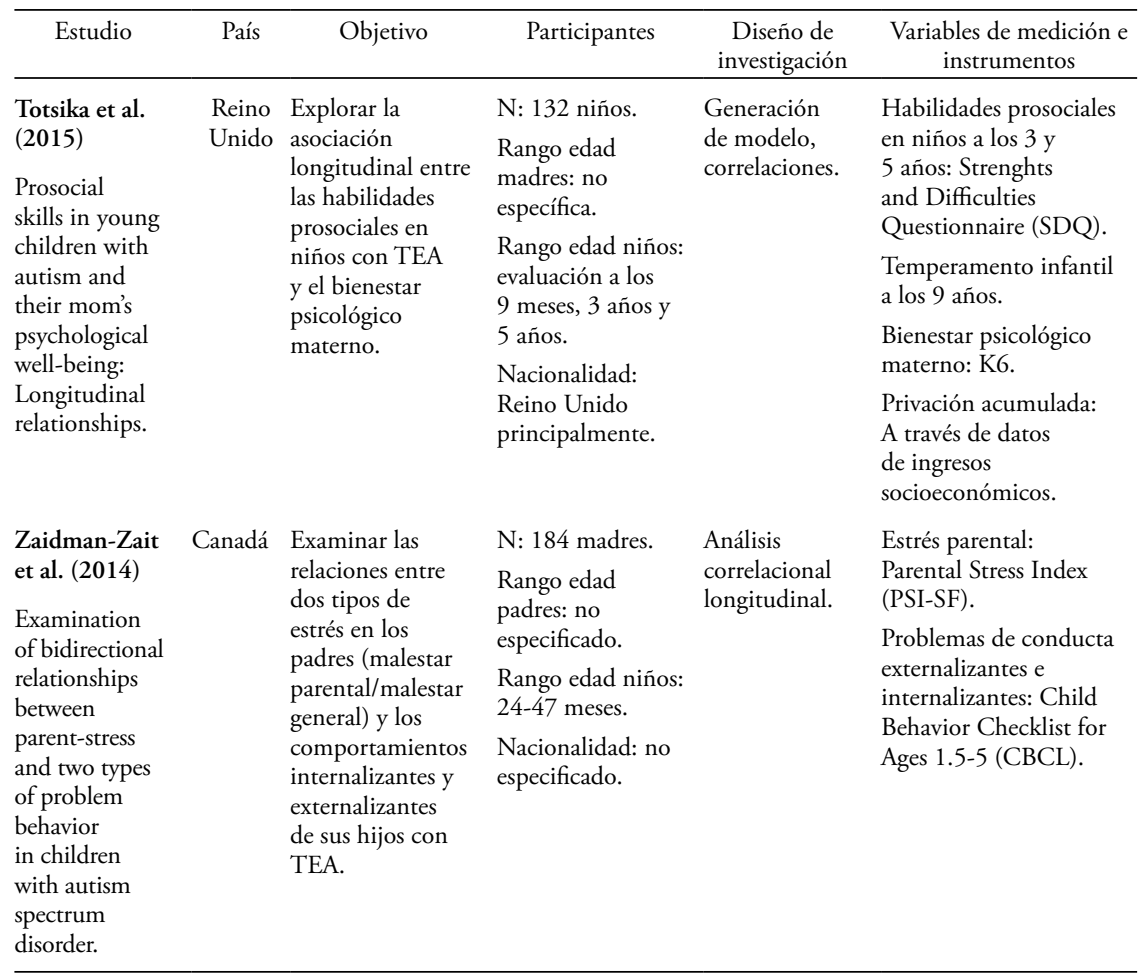

\section{Salud mental general}

En cuanto a los resultados de las investigaciones analizadas respecto a salud mental, cabe destacar que fue necesario crear una categoría denominada "salud mental general", puesto que los artículos revisados, utilizaron la escala Depression Anxiety Stress Scales (DASS-21) que mide estrés, ansiedad y depresión de manera simultánea. Por tanto, en sus resultados, no diferenciaban cada uno de los conceptos. Según los creadores de esta escala, estos tres síndromes representan un continuo y existen causas comunes subyacentes. Si bien conceptualmente el estrés, ansiedad y depresión son muy distintas, en la práctica clínica 
e investigación tienden a sobreponerse (Lovibond \& Lovibond, 1995, citado en Antúnez \& Vinet, 2012). En contraposición a esto, Totsika et al. (2013), señalan que las dificultades psicológicas específicas (estrés, depresión y ansiedad) siguen trayectorias diferentes a lo largo del tiempo, por lo que sería necesario estudiarlos de manera separada.

Como se apreció en los resultados de diversas investigaciones, los padres de hijos con TEA reportan altos niveles de estrés, depresión y dificultades en su salud mental (Bendixen et al., 2011; Bennet et al., 2012; Minjarez, Mercier, Williams \& Hardan, 2012; Rivard, Terroux, Parent-Boursier \& Mercier, 2014) poniendo en evidencia a este grupo como más vulnerable a desarrollar alguna psicopatología. Lo anterior se vuelve relevante pues, de presentar algunas de estas problemáticas, podrían verse afectadas otras áreas de sus vidas. Según Athari, Ghaedi, y Mohd Kosnin (2013), los padres de hijos con TEA se ven más expuestos a aumentar la severidad de los síntomas de sus hijos, afectando negativamente en el desarrollo de ellos. También, podría desencadenarse un peor funcionamiento a nivel familiar (Jellet, Wood, Giallo \& Seymour 2015), sentirse restringidos en las actividades familiares diarias (BenSasson, Soto, Martínez-Pedraza \& Carter, 2013) y en la percepción de que sus hijos presenten sintomatología más severa de lo que es en realidad (Bennet et al., 2012).

Cuando se observa qué características del niño con TEA o factores podrían mediar en un aumento de los problemas de salud mental de estos padres, las investigaciones analizadas indican que esta podría verse afectada por distintas variables. En cuanto a las características propias del TEA en sus hijos, las investigaciones mostraron que la severidad de los síntomas de autismo (Jellet et al., 2015), los problemas de conducta (Zaidman-Zait et al., 2014), algunos problemas sensoriales específicos (Ben-Sasson et al., 2013), el funcionamiento intelectual y la edad en que se diagnostica el trastorno (Rivard et al., 2014), podrían influir en el aumento o disminución en los niveles de dificultad observados en la salud mental de los padres.

Otros factores que se pudieron examinar y que también impactarían en su salud mental, serían la percepción de sentirse fatigados 
(Giallo Wood, Jellett \& Porter, 2013), los ingresos económicos familiares (Athari et al., 2013), el momento y la manera en que se recibe el diagnóstico, el momento en que se reciben los servicios de ayuda y los tipos de servicios obtenidos (Rivard et al., 2014).

\section{Estrés}

Respecto a los estudios sobre estrés, cabe destacar dos elementos que fueron considerados importantes. En primer lugar, todos los padres incluidos en las diversas muestras presentaban niveles altos y significativos de estrés. Respecto a esto, Wong, Seltzer, Greenberg y Hong (2012) seńalan que el alto estrés de estos padres sería permanente a lo largo de su vida, lo que pone en relieve la importancia de la prevención y tratamiento del estrés durante la infancia de sus hijos con el fin de no perpetuar estados de malestar por más tiempo. En segundo lugar, seis de los nueve artículos dentro de la categoría de estrés consistían en investigaciones que medían esta variable a partir de intervenciones que buscaban promover el desarrollo y mejores resultados en el niño con TEA a partir del entrenamiento de sus padres. Si bien el foco principal de estas intervenciones no se encontraba en promover la salud mental de estos padres, sí hipotetizaban que podría existir una mejoría en los niveles de estrés. Como resultado, los estudios de Strauss et al. (2012), Minjarez et al. (2012) y Bendixen et al. (2011), no mostraron una mejoría significativa en estos niveles, a pesar de que sí se observó mejoría en los niños. Probablemente la mantención del estrés, podría deberse a la alta demanda en tiempo que requería la intervención (Strauss et al., 2012) o podría depender de si era la madre o el padre quien recibía la intervención (Bendixen et al., 2011).

Se encontraron dos estudios que realizaban intervenciones enfocadas en el niño en donde se observaron resultados favorables en términos de salud mental en los padres. El primero fue realizado por Estes et al. (2014), el cual se caracterizó por implementar una intervención a las madres al poco tiempo de la confirmación diagnóstica de TEA en su hijo. Si bien estos resultados positivos podrían deberse a que las madres de esta muestra han estado expuestas a altos niveles de estrés 
por un período de tiempo más corto que las madres de otras investigaciones, este estudio mostraría que el acompañamiento psicoeducativo posterior a la confirmación diagnóstica ayudaría a reducir el estrés y a sobrellevar el difícil proceso que se inicia. Lo que permite sugerir que mientras antes los padres reciban ayuda, aunque sea dirigida al desarrollo del niño, mejores podrían ser los resultados en la disminución del estrés que ellos experimentan.

El segundo estudio realizado por Krakovich McGrew, Yu, y Ruble (2016), que mostró resultados favorables en los participantes fue una intervención que se enmarcó dentro del contexto escolar, en nińos con TEA mayores en edad que en la investigación de Estes y su equipo (2014). Tras la aplicación, se observó que el participar de esta intervención y presentar una mejor alianza entre padres y profesores se correlacionaba con menor nivel de estrés, tanto en relación con la percepción de su hijo como en su rol de padres. Estos hallazgos demuestran cómo el espacio y ayuda escolar puede ser una herramienta importante para disminuir el estrés de estos padres, y tiene el potencial adicional de reducir las disparidades en el acceso a estas instancias de apoyo requeridas por familias de entornos menos favorecidos.

En este sentido, pareciera interesante considerar que si se desean realizar terapias o intervenciones en donde el foco son los resultados del niño, también es posible que estas impacten en la salud mental de los padres, logrando una disminución en el nivel de estrés. Para esto, los hallazgos de todas estas investigaciones nos permiten mencionar las siguientes sugerencias como relevantes al momento de planificar estas intervenciones: 1) la aplicación de psicoeducación posterior al diagnóstico de TEA mostraría positivos resultados al favorecer la disminución de estrés; 2) el tiempo que requiera (en términos de horas semanales) no debiese ser experimentado por los padres como una demanda o exigencia extra a su diario vivir, causando así mayor estrés; 3) es relevante evaluar si será la madre o padre quien recibirá el entrenamiento, pues según Bendixen et al. (2011), en su estudio se observaron diferencias entre la madre y el padre en cuanto al estrés y la adaptabilidad, lo que sugiere que las estrategias de capacitación pueden necesitar adaptarse 
para reflejar el género y los roles de los padres; y 4) el espacio escolar y la alianza entre padres y profesores aparece como una valiosa alternativa para la aplicación de estrategias psicoeducativas, que a su vez, ayudarían a los padres a sentirse mejor respecto a ellos mismos y sus hijos.

\section{Ansiedad}

En cuanto al concepto de ansiedad, ningún artículo cumplió con los criterios para ser incluido. Lo anterior va en concordancia con lo seńalado por Conner, Maddox y White (2013), quienes refieren que esta temática se ha estado explorando recientemente y quizá, debido a esto, se comprenda la escasa cantidad de estudios en padres de niños con TEA. No obstante, los pocos estudios que han evaluado ansiedad han sido en muestras de padres de preadolescentes y adolescentes con TEA (Ooi et al., 2016; Conner et al., 2013; Uljarevic et al., 2016). En este sentido, resultaría interesante el estudio de esta variable en padres de individuos con TEA a lo largo del ciclo vital de sus hijos, pudiendo observar si existen variaciones dependiendo de la etapa del desarrollo.

\section{Depresión}

Respecto a depresión, la investigación de Bennet et al. (2012) puso en relieve la importancia de cómo la depresión materna puede influir en la forma en que las madres informan acerca de los comportamientos de sus niños con TEA y cómo los resultados de los cuestionarios pueden verse más afectados que los de las entrevistas semiestructuradas. Acorde con este estudio, Conner et al. (2013) también sugieren que otras variables de la salud mental, como la ansiedad, influirían en el reporte que dan los padres de sus hijos con TEA, pues en su investigación, los padres que manifestaron síntomas de ansiedad más altos también reportaron más sintomatología en sus hijos adolescentes. Siendo así necesario al momento de las evaluaciones clínicas y futuros estudios, el reporte de varios informantes, tales como padres, profesores, especialistas, entre otros. 


\section{Bienestar psicológico}

En cuanto a bienestar, en los dos artículos que se incluyeron, el concepto variaba dependiendo de lo que quisieran observar los investigadores, lo que concuerda con Winefield et al. (2012, citado en Samadi, Mcconkey \& Bunting, 2014), respecto a que no existe una definición consensuada de lo que comprende el bienestar psicológico y ningún instrumento estandarizado para medir este tipo de bienestar.

En otras investigaciones, el concepto incluía una variable psicológica, como estrés o depresión, y añadían otros factores como apoyo social (Smith, Greenberg \& Seltzer, 2012), esperanza y expectativas sobre el futuro de su hijo (Faso, Neal-Beevers \& Carlson, 2013) y satisfacción con la crianza (Samadi et al., 2014). Esto pone en relieve la necesidad de una conceptualización más específica y clara sobre bienestar, lo que podría permitir aunar los criterios y los resultados de la medición de esta variable.

\section{Intervenciones que promueven la salud mental de padres}

Para esta revisión, solo un artículo cumplía con los criterios de inclusión. Esta investigación fue realizada por Feinberg et al. (2014) y consistía en una breve intervención cognitiva conductual llamada "Problem Solving Education" (PSE), que buscaba disminuir el estrés y síntomas depresivos en las madres durante el período inmediatamente posterior al diagnóstico de un nińo con TEA. Esta intervención de modalidad individual, consistía en ayudar a los participantes a través de diferentes pasos: primero, identificar los sentimientos asociados a un problema específico; en segundo lugar, cambiar el enfoque del participante de los sentimientos a las soluciones que resuelven el problema; y por último, crear un plan de acción y generar pasos específicos para lograr la solución. El lugar utilizado para las sesiones podía ser la casa o la locación que las madres decidieran y su duración fue de seis sesiones de 30 a 45 minutos, una vez a la semana (un mes y medio en total).

Para esta investigación el grupo control consistía en madres de niños con TEA que recibían cuidado habitual, es decir, los servicios 
comunitarios que se ofrecen para niños con este trastorno. Como resultado, se observó que las madres del grupo PSE mostraron significativamente menos probabilidades de presentar estrés parental y síntomas depresivos clínicamente significativos en comparación con las madres que recibieron cuidados habituales, además de reportar un aumento en las habilidades sociales de afrontamiento. Con estos hallazgos, los investigadores sugieren que intervenciones como estas pueden tener un lugar en la práctica clínica. Asimismo, y considerando que los niños muy pequeños pasan más tiempo con sus padres y menos tiempo en la escuela, la participación de los padres en el plan de tratamiento del niño es esencial.

\section{Discusión}

En términos de las características de los estudios incluidos en esta revisión se observa que la mayoría de los artículos utilizaron exclusivamente metodología cuantitativa, especialmente autorreportes. Esto último, es considerado por algunos autores (Athari et al., 2013; Jellet et al., 2015; Krakovich et al., 2016; Totsika et al., 2015; Zaidman-Zait et al., 2014) como una limitación, ya que como los resultados se basaban en los reportes de los padres y las madres, y estos podrían verse influenciados por distintos factores que estuvieran afectando a los individuos al momento del estudio, pudiendo sub o sobre reportar sus propios niveles de malestar o las conductas de sus hijos (Bennet et al., 2012). Para esto, sugieren la importancia de complementar estos estudios con métodos cualitativos y de observación, con el fin de obtener una mejor comprensión y conocimiento de los fenómenos (Bennet et al., 2012).

Respecto a los focos de estudio, la mitad evaluó madres y la otra mitad a ambos padres. Esto podría deberse a que, a lo largo del tiempo, han sido las madres las que han puntuado mayores niveles de estrés y depresión en los estudios, volviéndose ellas de mayor interés para las investigaciones pues serían las que están más involucradas en el cuidado del niño. Siendo en la mayoría de los casos, la cuidadora principal, 
tornándose más susceptibles a verse afectadas por la conducta de su hijo (Jones, Totsika, Hastings \& Petalas 2013).

En cuanto a las investigaciones que han realizado intervenciones que buscan promover la salud mental en padres de niños con TEA, solo se analizó un artículo (Feinberg et al., 2014). Este resultado podría deberse a los criterios utilizados en esta revisión, especialmente el relacionado con la edad de los niños (de cero a nueve años), pues, en una revisión sobre intervenciones enfocadas en promover la salud mental en padres de hijos con TEA, la cual consideró un criterio de inclusión más amplio (Da Paz \& Wallander, 2016), se dio cuenta de 13 estudios que implementaban intervenciones. Los otros estudios, incluían a padres de hijos en diferentes etapas del desarrollo. Al observar que dentro de la presente revisión, seis artículos consistían en intervenciones enfocadas en el resultado del niño (y que fueron incluidas ya que cumplían el criterio de evaluar salud mental), se podría pensar que, durante la infancia, el foco de las intervenciones se encuentra en la psicoeducación a los padres con el objetivo de estimular y mejorar tanto las conductas como el desarrollo del niño, y no en el impacto psicológico que trae para los padres el diagnóstico de su hijo durante los primeros ańos. Sin embargo, dado los altos niveles de problemas en salud mental que mostraron los padres de las investigaciones presentadas anteriormente, el intervenir en la salud mental de estos padres desde que se confirma el diagnóstico pareciera ser otro foco relevante. Asimismo, sería importante considerar que tanto las intervenciones dirigidas al niño como aquellas enfocadas en la salud mental de sus padres podrían desarrollarse de manera simultánea e integral.

En cuanto a la modalidad de la intervención, Feinberg et al. (2014), implementaron un programa con foco individual, el cual tuvo resultados favorables al observar que las madres intervenidas mostraron significativamente menos probabilidades de presentar estrés parental y síntomas depresivos. Ante esto, en la revisión de Da Paz y Wallander (2016), se observó que tanto las intervenciones grupales como las individuales aparecían como igualmente efectivas. Si bien esto no es concluyente y requiere de más estudios. No obstante, permite pensar 
que el abordaje de las intervenciones que promuevan el bienestar de estos padres se pueda realizar ya sea de forma tanto individual como grupal, y que no habría una modalidad mejor que otra. Esto resulta interesante pues, dependiendo de las características de los padres, se les podría ofrecer distintas formas de intervención, pudiendo acceder a la que más se adecue a ellos.

Otro punto interesante, es que la intervención de Feinberg et al. (2014) utilizaba un enfoque cognitivo conductual para así disminuir el estrés y síntomas depresivos. Según el estudio de Da Paz y Wallander (2016), cuando las intervenciones dirigidas a padres de hijos con TEA se centraban en el "crecimiento personal", incluyendo variables como aceptación, mindfulness o gratitud, tendían a presentar grandes índices de deserción por parte de los participantes en el proceso. En cambio, las intervenciones enfocadas en la reducción de los síntomas, mostraban una permanencia en la asistencia de sus usuarios. Esto podría sugerir, que los padres dedicaban mayor tiempo a los tratamientos que estaban enfocados directamente en sus síntomas de malestar que en aquellos que tenían la intención de cambiar su perspectiva en situaciones difíciles (Da Paz \& Wallander, 2016). En esta misma línea, Romero-Martínez et al. (2017), en una reciente investigación sobre una intervención cognitivo conductual con padres de personas con TEA quienes habían cuidado a sus hijos por un período aproximado de 14 años, plantean que en forma posterior a la intervención que consistió en la aplicación de una serie de test sobre evaluación neuropsicológica, atención, memoria, lenguaje, y memoria ejecutiva, los padres tendrían una "mejor atención selectiva, memoria a corto y largo plazo en relación con las palabras cuentos e imágenes, es decir, flexibilidad cognitiva y técnicas de planificación, sin embargo, no se observan cambios con relación al estado cognitivo general" (RomeroMartínez et al. 2017, p. 2). Si bien este enfoque requiere de mayor investigación, mostraría que el modelo cognitivo conductual sería bastante efectivo y una alternativa considerable para promover la salud mental en estos padres. 


\section{Conclusiones}

A modo de conclusiones, el ser padres de niños con TEA es una experiencia compleja no solo por lo difícil de las características de este cuadro, sino también por los efectos adversos que puede generar en su salud mental. Si bien este trastorno es crónico y sus características permanecen a lo largo de la vida, la infancia presenta desafíos y aspectos que son particulares de esta etapa de desarrollo. Por lo que, conocer qué ocurre en este período con estos padres, podría ayudarlos a prevenir o disminuir problemáticas futuras. Es a partir de esto, que diversas investigaciones han buscado conocer los efectos, relaciones y/o factores que median en la salud mental de los padres a partir de la experiencia de tener un hijo con TEA.

Cabe mencionar que, aunque la literatura internacional ha aportado con importantes avances y hallazgos, pareciera que esta temática aún muestra un gran campo de estudio para seguir profundizando. En este sentido, los hallazgos obtenidos en los estudios internacionales se vuelven de gran importancia, dado que no se observaron investigaciones nacionales que cumplieran con los criterios de esta búsqueda. Esto permite considerar que, si los anteriores estudios fueran replicados en Chile, sería necesario tomar en cuenta las limitaciones de estas investigaciones y pensar en las características socioculturales particulares de este país. Así por ejemplo, las características de las muestras son principalmente madres y padres de países con tipos de salud, educación e ingresos económicos diferentes al de Chile. En este sentido, y como se mencionó anteriormente el gasto en salud a nivel nacional está por debajo del de otros países de la Organización para la Cooperación y el Desarrollo Económicos [OCDE]. Considerando lo anterior, es posible hipotetizar que la mayoría de los gastos en terapia para el niño sería costeada principalmente por los padres, por tanto, el contexto socioeconómico de los padres chilenos de niños con TEA, podría ser diferente al de los padres de los estudios incluidos en este trabajo. Esto implica un escenario distinto en cuanto a la aplicación de intervenciones dirigidas a estos padres, pues las características de ellas tendrían que ajustarse a 
la realidad nacional lo que incluye tanto los presupuestos para la salud pública como los ingresos económicos de los padres.

Parece pertinente considerar que, si bien es necesario el estudio de los padres de niños con TEA, igual de relevante es comenzar a generar un panorama global de cómo se da este trastorno en Chile, partiendo, como línea base, por un registro de personas diagnosticadas con TEA. Además, de lograr caracterizar a estos individuos y sus familias, se hace necesario conocer cómo acceden a la salud, cómo es el proceso diagnóstico, el tipo de intervenciones que reciben, entre otros.

De igual forma, es importante destacar que estas áreas de estudio requerirían de aproximaciones metodológicas que permitan conocer los fenómenos de la manera más completa pues, como se observó en algunos estudios incluidos en esta revisión, hubo diversas limitaciones metodológicas que restringieron una comprensión más acabada o compleja de los resultados $\mathrm{Al}$ respecto, es posible mencionar algunas sugerencias para tener presente en futuras investigaciones. Primero, se hace necesario definiciones claras de los conceptos a utilizar, de modo que permitan entender a qué fenómenos se están haciendo alusión y así compararlos más fácilmente con otros resultados que utilicen la misma variable. En segundo lugar, el hecho de encontrar solo investigaciones cuantitativas, resalta la necesidad de incorporar estudios cualitativos que incorporen estrategias metodológicas tales como la observación participante, entrevistas individuales, grupos focales, análisis de caso, entre otras, las que permitan un acercamiento a la experiencia subjetiva de los padres. En tercer lugar, el poder dar cuenta de las características de estos padres y sus familias serían valiosos hallazgos para ser tomados en consideración al momento de generar planes de intervención más acordes a las necesidades tanto de los niños como de sus padres.

Para finalizar, esta revisión se presentan diversas limitaciones. En este sentido, es posible señalar que los conceptos utilizados para entender "salud mental", es decir, estrés, ansiedad, depresión y bienestar pudieron generar un sesgo que no permite una comprensión más amplia de la temática de estudio. A su vez, el centrarse en distintos elementos tampoco permite profundizar en cada uno de ellos. Asimismo, 
la selección de bases de datos y revistas científicas contribuyó a que otros artículos con interesantes aportes que no cumplían los criterios de selección de las bases de datos, hayan quedado fuera.

De igual forma, las edades requeridas generaron una limitación en esta revisión, pues si bien existen investigaciones que incluyen niños entre cero a nueve años como parte de una muestra con edades más variadas, esos estudios quedaron excluidos pues los rangos de edad eran muy amplios. Quizás, de haber sido incorporados, las conclusiones habrían variado. Además, al requerirse que los estudios explicitaran los rangos de edad, varios de ellos quedaron fuera, pues muchos solo indicaban la media de estas edades.

Es considerando todo lo anterior, que se sugiere ampliar las investigación de esta temática en Chile, comenzando por cambiar paulatinamente el foco desde los problemas o causas de este trastorno en nińos y adolescentes a las experiencias de sus padres no solo en lo que se refiere a su salud mental e intervenciones, sino que también en otros elementos relevantes que rodean este trastorno, como lo son el apoyo social, las experiencias de otras figuras significativas y cuidadores, las transformaciones en las dinámicas familiares, la visión de profesionales que trabajan con estos usuarios y sus familias, la creación de nuevas intervenciones, entre otras. Asimismo, y tal como sugieren RomeroMartínez et al. (2017) se hace imprescindible tomar en consideración el impacto de la discapacidad cognitiva en las habilidades para proporcionar un cuidado de calidad a largo plazo de estos padres, lo que implica establecer estrategias e intervenciones de apoyo psicosocial que les permitan un respiro para reducir el estrés a través de programas que aumenten sus capacidades cognitivas, si pensamos en mejorar la salud mental y bienestar de estos padres. Toda esta información aportaría valiosamente en la ayuda y comprensión del TEA, en favor de quienes lo vivencian directamente, en sus padres, sus familias, los profesionales y la sociedad en general. 


\section{Referencias}

Agency for Healthcare Research and Quality (2011). Research reviews: Comparative effectiveness of therapies for children with autism spectrum disorders. U.S. Department of Health and Human Services, Agency for Healthcare Research and Quality. Recuperado de http://www.effectivehealthcare.ahrq.gov/ehc/ products/106/651/Autism Disorder exec-summ.pdf

Aguaded, M. \& Almeida, N. (2016). El enfoque neuropsicológico del autismo: reto para comprender, diagnosticar y rehabilitar desde la atención temprana. Revista Chilena de Neuropsicología, 11(2), 34-39.

Al-Farsi, O., Al-Farsi, Y., Al-Sharbati, M. \& Al-Adawi, S. (2016). Stress, anxiety, and depression among parents of children with autism spectrum disorder in Oman: A case-control study. Neuropsychiatric Disease and Treatment, 12, 1943-1951. https://doi.org/10.2147/NDT.S107103

AlHorany, A., Younis, N., Bataineh, M. \& Hassan, S. (2013). Do Mothers of Children with Autism are at Higher Risk of Depression? A Systematic Review of Literature. Life Science Journal, 10(1), 4303-4308. Recuperado de https://www. researchgate.net/publication/286050459_Do_mothers_ of_autistic_children_are_at_higher_risk_of_depression_A_ systematic_review_of_literature

American Psychiatric Association (APA). (2000). Diagnostic and statistical manual of mental disorders (4th ed.). Washington, D.C.: American Psychiatric Association.

American Psychiatric Association (2013). Autism spectrum disorder. Recuperado de https:/www.psychiatry.org/File\%20Library/ Psychiatrists/Practice/DSM/APA_DSM-5-Autism-SpectrumDisorder.pdf

Athari, P., Ghaedi, L. \& Mohd Kosnin, A. (2013). Mothers' depression and stress, severity of autism among children and family income. International Journal of Psychological Research, 6(2), 98-106. https://doi.org/10.21500/20112084.691 
Bekhet, A. K. (2014). Self-assessed health in caregivers of persons with autism spectrum disorder: Associations with depressive symptoms, positive cognitions, resourcefulness, and well-being. Perspectives in Psychiatric Care, 50(3), 210-217. https://doi. org/10.1111/ppc. 12046

Bekhet, A., Johnson, N. \& Zauszniewski, J. (2012). Resilience in family members of persons with autism spectrum disorder: A review of the literature. Issues in Mental Health Nursing, 33(10), 650-656. https://doi.org/10.3109/01612840.2012.671441

Bekhet, A. \& Zauszniewski, J. (2013). Psychometric assessment of the depressive cognition scale among caregivers of persons with autism spectrum disorder. Archives of Psychiatric Nursing, 27(2), 96-100. https://doi.org/10.1016/j.apnu.2012.10.004

Ben-Sasson, A., Soto, T., Martínez-Pedraza, F. \& Carter, A. (2013). Early sensory over-responsivity in toddlers with autism spectrum disorders as a predictor of family impairment and parenting stress. Journal of Child Psychology and Psychiatry and Allied Disciplines, 54(8), 846-853. https://doi.org/10.1111/jcpp.12035

Bendixen, R., Elder, J., Donaldson, S., Kairalla, J., Valcante, G. \& Ferdig, R. (2011). Effects of a father-based in-home intervention on perceived stress and family dynamics in parents of children with autism. American Journal of Occupational Therapy, 65(6), 679-687. https://doi.org/10.5014/ajot.2011.001271

Bennett, T., Boyle, M., Georgiades, K., Georgiades, S., Thompson, A., Duku, E., ... Szatmari, P. (2012). Influence of reporting effects on the association between maternal depression and child autism spectrum disorder behaviors. The Journal of Child Psychology and Psychiatry, 53(1), 89-96. https://doi. org/10.1111/j.14697610.2011.02451.x

Berríos, P., Catalán, F., Muñoz, C., Maureira, M. \& Santibáñez, A. (2012). Comparación de habilidades de teoría de la mente entre sujetos con sindrome de asperger y sujetos con desarrollo tipico. (Tesis Pregrado Fonoaudiología, Universidad de Chile, Chile). Recuperado de http://www.repositorio.uchile.cl/handle/2250/114908 
Bonis, S. \& Sawin, K. (2016). Risks and protective factors for stress self-management in parents of children with autism spectrum disorder: An integrated review of the literature. Journal of Pediatric Nursing, 31(6), 567-579. https://doi.org/10.1016/j. pedn.2016.08.006

Cabezas, H. (2001). Los padres del niño con autismo. Actualidades Investigativas en Educación, 1(2), 1-16. Recuperado de www. redalyc.org/articulo.oa?id $=44710202$

Cachia, R., Anderson, A. \& Moore, D. (2016). Mindfulness, stress and well-being in parents of children with autism spectrum disorder: A systematic review. Journal of Child and Family Studies, 25(1), 1-14. https://doi.org/10.1007/s10826-015-0193-8

Carrera, M. (2012-12). El cuerpo en el autismo. (Memoria Pregrado Psicología, Universidad de Chile, Chile). Recuperado de http:// www.repositorio.uchile.cl/handle/2250/116972

Centers for Disease Control and Prevention (CDC). (2014). Autism Spectrum Disorder: Data and Statistics. Recuperado de https:// www.cdc.gov/ncbddd/autism/data.html

Conner, C., Maddox, B. \& White, S. W. (2013). Parents state and trait anxiety: Relationships with anxiety severity and treatment response in adolescents with autism spectrum disorders. Journal of Autism and Developmental Disorders, 43(8), 1811-1818. https://doi.org/10.1007/s10803-012-1728-0

Cridland, E., Jones, S., Magee, C. \& Caputi, P. (2014) Family focused autism spectrum disorder research: a review of the utility of family systems approaches. Autism, 18(3), 213-222. https:// doi.org/10.1177/1362361312472261

Croen L., Grether J., Yoshida C., Odouli R. \& Hendrick V. (2011). Antidepressant use during pregnancy and childhood autism spectrum disorders. Archives of General Psychiatry, 68(11), 11041112. https://doi.org/10.1001/archgenpsychiatry.2011.73

Da Paz, N. \& Wallander, J. (2017). Interventions that target improvements in mental health for parents of children with autism spectrum disorders: A narrative review. Clinical Psychology Review, 51, 1-14. https://doi.org/10.1016/j.cpr.2016.10.006 
Dababnah, S. \& Parish, S. (2015). A comprehensive literature review of randomized controlled trials for Parents of young children with autism spectrum disorder. Journal of Evidence-Informed Social Work, 13(3), 277-292. https://doi.org/10.1080/2376140 7.2015.1052909

Estes, A., Vismara, L., Mercado, C., Fitzpatrick, A., Elder, L., Greenson, J.,... Rogers, S. (2014). The impact of parentdelivered intervention on parents of very young children with autism. Journal of Autism and Developmental Disorders, 44, 353365. https://doi.org/10.1007/s10803-013-1874-z

Faso, D., Neal-Beevers, A. \& Carlson, C. (2013). Vicarious futurity, hope and well-being in parents of children with autism spectrum disorder. Research in Autism Spectrum Disorders, 7(2), 288-297. https://doi.org/10.1016/j.rasd.2012.08.014

Feinberg, E., Augustyn, M., Fitzgerald, E., Sandler, J., Ferreira-Cesar Suarez, Z., Chen, N. \& Silverstein, M. (2014). Improving maternal mental health after a child's diagnosis of autism spectrum disorder: results from a randomized clinical trial. JAMA Pediatrics, 168(1), 40-46. https://doi.org/10.1001/ jamapediatrics.2013.3445

Fernández, M. (2016). Acompañamiento a través del arte: una intervención de arteterapia con un niño con trastorno del espectro-autista. (Postítulo de Especialización en Terapia de Arte mención Arteterapia, Universidad de Chile, Chile). Recuperado de http:// repositorio.uchile.cl/handle/2250/143181

Geoffray, M., Thevenet, M. \& Georgieff, N. (2016). News in early intervention in autism. Psychiatria Danubina, 28(1), 66-70. Recuperado de https:/www.ncbi.nlm.nih.gov/ pubmed/27663808

Giallo, R., Wood, C., Jellett, R. \& Porter, R. (2013). Fatigue, wellbeing and parental self-efficacy in mothers of children with an Autism Spectrum Disorder. Autism, 17(4), 465-480. https://doi. org/10.1177/1362361311416830 
Golfenshtein, N., Srulovici, E. \& Medoff-Cooper, B. (2015). Investigating parenting stress across pediatric health conditions: A systematic review. Issues in Comprehensive Pediatric Nursing, 14, 1-49. https://doi.org/10.3109/01460862.2015.1078423

Gomes, P., Lima, L., Bueno, M., Araújo, L. \& Souza, N. (2015). Autism in Brazil: A systematic review of family challenges and coping strategies. Jornal de Pediatria, 91(2), 111-121. https:// doi.org/10.1016/j.jped.2014.08.009

Hayes, S. \& Watson, S. (2013). The impact of parenting stress: A meta-analysis of studies comparing the experience of parenting stress in parents of children with and without autism spectrum disorder. Journal of Autism and Developmental Disorders, 43(3), 629-642. https://doi.org/10.1007/s10803-012-1604-y

Hernández, P. (2008). Impacto psicosocial en familias con un hijo con autismo. Relatos autobiográficos de seis madres de distinto nivelsocioeconómico. (Tesis Pregrado Trabajo Social, Universidad Academia de Humanismo Cristiano, Chile). Recuperado de bibliotecadigital.academia.cl/bitstream/handle/123456789/884/TTRASO $\% 20240$.pdf?sequence $=1$ \&isAllowed $=y$

Irarrázaval M., Brokering W. \& Murillo G. (2005). Autismo: una mirada desde la psiquiatría de adultos. Revista Chilena de Neuropsiquiatría, 43(1), 51-60. https://doi.org/10.4067/S0717-9227 2005000100007

Jellett, R., Wood, C., Giallo, R. \& Seymour, M. (2015). Family functioning and behaviour problems in children with autism spectrum disorders: The mediating role of parent mental health. Clinical Psychologist, 19(1), 39-48. https://doi.org/10.1111/ cp. 12047

Jones, L., Totsika, V., Hastings, R. \& Petalas, M. (2013). Gender differences when parenting children with autism spectrum disorders: A multilevel modeling approach. Journal of Autism and Developmental Disorders, 43(9), 2090-2098. https://doi.org/ 10.1007/s10803-012-1756-9 
Karst, J. \& van Hecke, A. V. (2012). Parent and family impact of autism spectrum disorders: A review and proposed model for intervention evaluation. Clinical Child and Family Psychology Review, 15(3), 247-277. https://doi.org/10.1007/ s10567-012-0119-6

Krakovich, T., McGrew, J., Yu, Y. \& Ruble, L. (2016). Stress in parents of children with autism spectrum disorder: An exploration of demands and resources. Journal of Autism and Developmental Disorders, 46(6), 2042-2053. https://doi.org/10.1007/s10803016-2728-2

Kuusikko-Gauffin, S., Pollock-Wurman, R., Mattila, M., Jussila, K., Ebeling, H., Pauls, D. \& Moilanen, I. (2013). Social anxiety in parents of high-functioning children with autism and Asperger syndrome. Journal of Autism and Developmental Disorders, 43(3), 521-529. https://doi.org/10.1007/s10803-012-1581-1

Lee, J. (2013). Maternal stress, well-being, and impaired sleep in mothers of children with developmental disabilities: A literature review. Research in Developmental Disabilities, 34(11), 42554273. https://doi.org/10.1016/j.ridd.2013.09.008

Llantén, K. \& Valencia, C. (2001). Autismo infantil desde una perspectiva psicoanalitica. Frances Tustin. (Tesis Pregrado Psicología, Universidad de Chile, Chile). Recuperado de http://repositorio. uchile.cl/handle/2250/136917

Ministerio de Salud (MINSAL). (2011). Guía de Práctica Clínica. Detección y diagnóstico oportuno de los trastornos del espectro autista. Santiago, Chile.

Ministerio de Salud (MINSAL). (2014). Norma técnica para la supervisión de niños y niñas de 0 a 9 años en la Atención Primaria de Salud. Programa Nacional de Salud de la Infancia. Santiago, Chile.

Minjarez, M., Mercier, E., Williams, S. \& Hardan, A. (2012). Impact of pivotal response training group therapy on stress and empowerment in parents of children with autism. Journal of Positive Behavior Interventions, 15(2), 71-78. https://doi. org/10.1177/1098300712449055 
Miranda, F. (2007). Intervención musicoterapéutica en un niño que presenta trastorno del espectro autista. (Tesis Postgrado en Terapia del Arte, mención en Musicoterapia, Universidad de Chile, Chile). Recuperado de http://www.repositorio.uchile.cl/handle/ 2250/101417

Morales, M., Solovieva, Y., Lázaro, E., Quintanar, L. \& Machinskaya, R. (2014). Análisis neuropsicológico y neurofisiológico en una niña con autismo: estudio longitudinal con resultados de intervención. Revista Chilena de Neuropsicología, 9(E2), 72-79. Recuperado de http://www.neurociencia.cl/dinamicos/ articulos/882648-rcnp2014v9ne2-5.pdf

Moya, M. (2016). El humor positivo y su vinculo con la creatividad y la flexibilidad cognitiva en el sindrome de Asperger. (Tesis Magíster en Estudios Cognitivos, Universidad de Chile, Chile). Recuperado de http://repositorio.uchile.cl/handle/2250/140645

Ooi, K., Ong, Y., Jacob, S. \& Khan, T. (2016). A meta-synthesis on parenting a child with autism. Neuropsychiatric Disease and Treatment, 12, 745-762. https://doi.org/10.2147/NDT. S100634

Organización Mundial de la Salud (s.f). Temas de Salud. Salud Mental. Recuperado de http://www.who.int/topics/mental_health/es/

Organización Mundial de la Salud (s.f). Temas de Salud. Depresión.

Recuperado de http://www.who.int/topics/depression/es/

Organización Mundial de la Salud (2013). El desarrollo del niño en la primera infancia y la discapacidad: un documento de debate. Ginebra: Organización Mundial de la Salud.

Organización Mundial de la Salud (2016). Preguntas y respuestas sobre los trastornos del espectro autista (TEA). Recuperado de http:// www.who.int/features/qa/85/es/

Organización para la Cooperación y el Desarrollo Económicos [OCDE]. (2015) Health at a Glance 2015, ¿Cómo compara Chile? París: OECD Publishing. Recuperado de https://www.oecd. org/chile/Health-at-a-Glance-2015-Key-Findings-CHILE-InSpanish.pdf 
Puglisevich, J. (2014). Trastornos del espectro autista: Consideraciones evolutivas y lineamientos psicoterapéuticos desde el enfoque constructivista evolutivo con base en modelos actuales de intervención. (Tesis Pregrado Psicología, Universidad de Chile, Chile). Recuperado de http://repositorio.uchile.cl/handle/2250/135467

Quijada, C. (2008). Espectro autista. Revista Chilena de Pediatría, 79(Supl. 1), 86-91. https://doi.org/10.4067/S0370-410620080 00700013

Rai, D., Lee, B., Dalman, C., Golding, J., Lewis, G. \& Magnusson, C. (2013). Parental depression, maternal antidepressant use during pregnancy, and risk of autism spectrum disorders: population based case-control study. Bmj, 346, f2059. https://doi.org/ 10.1136/bmj.f2059

Rivard, M., Terroux, A., Parent-Boursier, C. \& Mercier, C. (2014). Determinants of stress in parents of children with autism spectrum disorders. Journal of Autism and Developmental Disorders, 44(7), 1609-1620. https://doi.org/10.1007/s10803-013-2028-z

Roberts, A., Koenen, K., Lyall, K., Ascherio, A. \& Weisskopf, M. (2014). Women's posttraumatic stress symptoms and autism spectrum disorder in their children. Research in Autism Spectrum Disorders, 8(6), 608-616. https://doi.org/10.1016/j.rasd.2014.02.004

Romero-Martínez, A., Ruiz-Robledillo, N., Sariñana-González, P., de Andrés-García, S., Vitoria-Estruch, S. \& Moya-Albiol, L. (2017). A cognitive-behavioural intervention improves cognition in caregivers of people with autism spectrum disorder: A pilot study. Psychosocial Intervention, 26(3), 165-170. https:// doi.org/10.1016/j.psi.2017.06.002

Rother, E. (2007). Revisión sistemática x revision narrativa. Acta Paulista de Enfermagem, 20(2), v-vi. https://doi.org/10.1590/ S0103-21002007000200001

Saa, Y. (2014). La experiencia de Lucas. Musicoterapia con un niño del espectro autista. (Tesis Postgrado Terapia de Arte, mención Musicoterapia, Universidad de Chile, Chile). Recuperado de http:// www.repositorio.uchile.cl/handle/2250/116583 
Samadi, S. \& Mcconkey, R. (2014). The impact on Iranian mothers and fathers who have children with an autism spectrum disorder. Journal of Intellectual Disability Research, 58(3), 243254. https://doi.org/10.1111/jir.12005

Smith, L., Greenberg, J., y Seltzer, M. (2012). Social support and wellbeing at mid-fife among mothers of adolescents and adults with autism spectrum disorders. Journal of Autism and Developmental Disorders, 42(9), 1818-1826. https://doi.org/10.1007/ s10803-011-1420-9

Snow, M. \& Donnelly, J. (2016). Factors Mediating Dysphoric Moods and Help Seeking Behaviour Among Australian Parents of Children with Autism. Journal of Autism and Developmental Disorders, 46(6), 1941-1952. https://doi.org/10.1007/s10803016-2725-5

Soto, P. (2007). Atención conjunta y autismo. Estudio en niños preescolares entre 2 y 5 años de edad. (Tesis Magíster Psicología, mención Psicología Clínica Infanto Juvenil). Recuperado de http://www. repositorio.uchile.cl/handle/2250/106577

Strauss, K., Vicari, S., Valeri, G., D’Elia, L., Arima, S. \& Fava, L. (2012). Parent inclusion in early intensive behavioral intervention: The influence of parental stress, parent treatment fidelity and parentmediated generalization of behavior targets on child outcomes. Research in Developmental Disabilities, 33(2), 688-703. https:// doi.org/10.1016/j.ridd.2011.11.008

Tereucán, P. \& Treimún, N. (2016). Estrés parental y estrategias de afrontamiento en familias de niños(as) con trastorno del espectro autista. (Seminario Titulación Educación Diferencial, Universidad Austral de Chile, Chile). Recuperado de tesis.pucp.edu. pe/repositorio/handle/123456789/1659

Tobar, M. (2005). Resiliencia en familias con hijos autistas. (Tesis Pregrado Trabajo Social, Universidad Academia de Humanismo Cristiano, Chile). Recuperado de bibliotecadigital.academia.cl/ handle/123456789/1469 
Totsika, V., Hastings, R., Emerson, E., Lancaster, G., Berridge, D. \& Vagenas, D. (2013). Is there a bidirectional relationship between maternal well-being and child behavior problems in autism spectrum disorders? Longitudinal analysis of a populationdefined sample of young children. Autism Research, 6(3), 201-211. https://doi.org/10.1002/aur.1279

Totsika, V., Hastings, R., Emerson, E., Berridge, D. \& Lancaster, G. (2015). Prosocial skills in young children with autism and their mother's psychological well-being: Longitudinal relationships. Research in Autism Spectrum Disorders, 13-14, 25-31. https:// doi.org/10.1016/j.rasd.2015.01.001

Uljarevic, M., Carrington, S. \& Leekam, S. (2016). Effects of sensory sensitivity and intolerance of uncertainty on anxiety in mothers of children with autism spectrum disorder. Journal of Autism and Developmental Disorders, 46(1), 315-319. https://doi. org/10.1007/s10803-015-2557-8

Van Steijn, D., Oerlemans, A., Van Aken, M., Buitelaar, J. \& Rommelse, N. (2014). The reciprocal relationship of ASD, ADHD, depressive symptoms and stress in parents of children with ASD and/or ADHD. Journal of Autism and Developmental Disorders, 44(5), 1064-1076. https://doi.org/10.1007/s10803-013-1958-9

Whitmore, K. (2016). Respite care and stress among caregivers of children with autism spectrum disorder: An integrative review. Journal of Pediatric Nursing, 31(6), 630-652. https://doi. org/10.1016/j.pedn.2016.07.009

Wong, D., Seltzer, M., Greenberg, J. \& Hong, J. (2012). Stressful life events and daily stressors affect awakening cortisol level in midlife mothers of individuals with autism spectrum disorders. Aging and Mental Heatlh, 16(9), 939-949. https://doi.org/10.10 80/13607863.2012.688191

Zablotsky, B., Anderson, C. \& Law, P. (2013). The association between child autism symptomatology, maternal quality of life, and risk for depression. Journal of Autism and Developmental Disorders, 43(8), 1946-1955. https://doi.org/10.1007/s10803-012-1745-z 
Zaidman-Zait, A., Mirenda, P., Duku, E., Szatmari, P., Georgiades, S., Volden, J., ... Pathways in ASD Study Team. (2014). Examination of bidirectional relationships between parent stress and two types of problem behavior in children with autism spectrum disorder. Journal of Autism and Developmental Disorders, 44(8), 1908-1917. https://doi.org/10.1007/s10803-014-2064-3

Zalaquett, D., Schönstedt, M., Angeli, M., Herrrera, C. \& Moyano, A. (2015). Fundamentos de la intervención temprana en niños con trastornos del espectro autista. Revista Chilena de Pediatría, 86(2), 126-131. https://doi.org/10.1016/j.rchipe.2015.04.025

Zhou, T. \& Yi, C. (2014). Parenting styles and parents' perspectives on how their own emotions affect the functioning of children with autism spectrum disorders. Family Process, 53(1), 67-79. https:// doi.org/10.1111/famp. 12058

Recibido: 25 de mayo, 2018

Revisado: 14 de febrero, 2019

Aceptado: 11 de abril, 2019 